\title{
On the Foundations of Mathematical Modeling of Economy
}

\author{
A.A. PETROV \\ Computing Center of RAS, Russia
}

\section{INTRODUCTION}

The main issue of my report is economic sense of Conditions of Integrability of Consumers Demand Function. Generally speaking, these are the conditions of existence of consumption and price indices for separated groups of goods. In formal sense, integrability conditions are an analog of The Second Law of Thermodynamics but they are not always fulfilled. Shananin showed by means of numerical experiments with data on consumption statistics that integrability conditions were not fulfilled for a period of 19321935 when economic structures were being modified.

On the other hand, it is shown that if the consumer demand functions satisfy the integrability conditions, then the Leontieff description of multi-product producing system regulated by equilibrium market mechanisms can be aggregated by means of the consumption index (utility function) into a production function. The latter describes the way the production index depends on the primary resources used by the producing system.

The aggregated description expresses the equilibrium between macro-parts of the economy, namely the industry proposing consumer goods and nonproducing sector demanding these. The description is correct because it corresponds to a detailed equilibrium between supply and demand of separate products.

Thus, if the consumption index exists (and hence, the dual price index also exists), then the economy is organized well, and production and consumption are agreed. The cost law reveals not only in a number of elementary exchanges, but also in macro-exchanges between the macro-parts. Regulating financial mechanisms are effective.

Therefore, it is interesting to investigate the economic sense of integrability conditions. I shall use for my task a well-known Neoclassical Model of Consumer Demand.

\section{AGGREGATION OF INCOME DISTRIBUTION}

Let us consider a group of $m$ products. An arbitrary set of the products is denoted by $X=\left(X_{1}, X_{2}, \ldots, X_{m}\right)$, and the vector of the corresponding prices is denoted by $\mathbf{p}=\left(p_{1}, p_{2}, \ldots, p_{m}\right)$. Assume that $M$ social groups are selected in a society according to their stereotypes of consumer behavior. The stereotype of consumer behavior for the $\alpha$ th group is described by the problem on maximizing a positive uniform function $u_{\alpha}(X)$ 
subject to the budget constraint $(\mathbf{p}, X) \leq I_{\alpha}, X \geq 0$, where $I_{\alpha}$ is the income of the $\alpha$ th group used for consumption. We assume that the utility function $u_{\alpha}(X)$ belongs to the class $A_{m}$ and $u_{\alpha}(X)=0$ for $X \in$ $\partial R_{+}^{m}$.

If the normalized demand functions of the $\alpha$ th group is denoted by $y_{\alpha}(\mathbf{p})$ then its demand is given as $I_{\alpha} y_{\alpha}(\mathbf{p})$. Thus, the price index from the point of view of the $\alpha$ th group is determined using the formula

$$
q_{\alpha}(\mathbf{p})=\inf \left\{\frac{(\mathbf{p}, X)}{u_{\alpha}(X)} \mid X \geq 0, u_{\alpha}(X)>0\right\}
$$

and the total consumption fund of the society is given by $I=\sum_{\alpha=1}^{M} I_{\alpha}$.

According to the neoclassical theory, we assume that the way the income is distributed among the social groups depends on the prices p. Indeed, changes in price structure cause changes in social behavior of population. Real income, in particular, changes, and this results in migration from one social group to another. The distribution of income among the groups also changes.

The part of income of the $\alpha$ th social group [ $\left.\varphi_{\alpha}(\mathbf{p})\right]$ in the total consumption fund $I$ is given by $\varphi_{\alpha}(\mathbf{p})=$ $I_{\alpha} / I$, assuming that $\varphi_{\alpha}(\mathbf{p})$ are positively uniform functions.

Let us calculate the total consumer demand of the society $I y(\mathbf{p})$ which unites the demands of social groups. It is clear that $y(\mathbf{p})$ satisfies the separability conditions.

Proposition 1. The differential form of the demand can be represented as

$$
y(\boldsymbol{p}) d \boldsymbol{p}=\sum_{\alpha=1}^{M} \frac{\varphi_{\alpha}(\boldsymbol{p})}{q_{\alpha}(\boldsymbol{p})} d q_{\alpha}(\boldsymbol{p})
$$

The vector of price indices calculated from the points of view of various groups is denoted by $q(p)=$ $\left(q_{1}(p), q_{2}(\boldsymbol{p}), \ldots, q_{M}(\boldsymbol{p})\right)$, assuming that the system of functions $q(\boldsymbol{p})$ is functionally independent.
Proposition 2. Assume that there exists a consumption index $F(X)$ and price index $Q(p)$ belonging to the class $A_{m}$ and corresponding to the demand functions $y(\boldsymbol{p})$. Then there exists a function $\Phi(q)$ such that $Q(p)=\Phi(q(p))$ and

$$
\begin{gathered}
\varphi_{\alpha}(\boldsymbol{p})=\left(\frac{q_{\alpha}(\boldsymbol{p})}{\Phi(q(\boldsymbol{p}))}\right)\left(\frac{\partial \Phi}{\partial q_{\alpha}}\right)(q(\boldsymbol{p})), \\
\alpha=1, \ldots, M
\end{gathered}
$$

The interpretation of Proposition 1 complements that of equilibrium theory of aggregation. Now we have proved that for the integrability conditions to be satisfied for the demand functions $y(\mathbf{p})$, it is necessary that the distribution $\left\{\varphi_{\alpha}(\mathbf{p})\right\}$ of income among social groups depend implicitly on the prices, i.e. depend on the price indices $q(\mathbf{p})$ by which various social groups estimate the level of consumer prices. This implies that the distribution of income in the society should agree with the estimates of price level existing in the society. This can be interpreted in a logical manner. Selfregulating mechanisms for distributing income should work in the society. Thus, we see that mechanisms should exist for self-regulating economical processes and relations between economic agents. Economists associate them with market mechanisms. This, in particular, concerns Proposition 1. It is asserted that good markets cannot work in a normal way if labor market does not exist.

Thus assume that the distribution of income depends on the prices in terms of the indices $q(\mathbf{p})$, namely $\varphi_{\alpha}(\mathbf{p})=\delta_{\alpha}(q(\mathbf{p})), \alpha=1, \ldots, M$.

If the distribution of income can be represented like in Eq. (2) at some function $\Phi(q)$, then the differential form of demand $(y(\mathbf{p})$, dp) satisfies, clearly, the integrability conditions. In order for the price index $Q(\mathbf{p})=\Phi(q(\mathbf{p})) q(\mathbf{p})=\Phi(q(\mathbf{p}))$ to be continuous, convex and monotonously nondecreasing on $R_{+}^{m}$ for any $q(\mathbf{p})$ satisfying the same conditions, it is necessary and sufficient that the function $\Phi(q)$ also satisfies the same conditions for the economic indices. 
The function $\Phi(q)$ turns out to be connected with Bergsonian Function of Welfare. Let us consider the function dual to $\Phi(q)$, namely

$$
W(u)=\inf \left\{\frac{(q, u)}{\Phi(q)} q \geq 0, \quad \Phi(q)>0\right\}
$$

where $u=\left(u_{1}, u_{2}, \ldots, u_{M}\right)$ is the vector of utility functions of various social groups.

Proposition 3. Let $\Phi(q) \in A_{m}$. Put $\hat{u}_{\alpha}(q)=$ $(1 / \Phi(q))\left(\partial \Phi(q) / \partial q_{\alpha}\right), \alpha=1, \ldots, M$. Then $\hat{u}_{\alpha}(q)=$ $\left(\hat{u}_{1}(q), \hat{u}_{2}(q), \ldots, \hat{u}_{M(q)}(q)\right)$ is a solution to the optimization problem

$$
W(u) \Rightarrow \max \quad \text { subject to } \quad(q, u) \leq 1, \quad u \geq 0 .
$$

In economical theory, the function $W(u)$ is referred to as the Bergsonian welfare function. Let us consider how it is related to the consumption index $F(X)$.

Proposition 4. Let $\Phi(q) \in A_{m}$, and let $\left\{\varphi_{\alpha}(\boldsymbol{p})\right\}$ be defined by the formula Eq. (2). Then the consumption index $F(X)$ is not less than the optimal value of the functional in the equation

$$
\begin{aligned}
& W\left(u_{1}\left(X^{1}\right), u_{1}\left(X^{2}\right), \ldots, u_{M}\left(X^{M}\right)\right) \Rightarrow \max \\
& \text { subject to } \sum_{\alpha=1}^{M} X^{\alpha}=X, \quad X^{\alpha} \geq 0, \\
& \alpha=1, \ldots, M .
\end{aligned}
$$

If $X=I \cdot y(\mathbf{p})$ for some $\mathbf{p}>0$, then $F(X)$ is equal to the optimal value of the functional in Eq. (4).

Usually, political economy studies the problem on fair distribution of income in the society. Generally, a concept of fairness is proposed to which the distribution of income should correspond. The concept is expressed formally by the Bergson function. This is a specially constructed function whose maximum is attained just at the distribution of income corresponding the concept.
The Bergson function expresses a compromise between economical interests of social groups, and can be treated as a political "party program". It seems that the program can be prescribed directly by the functions $\delta_{\alpha}(q(\mathbf{p})), \alpha=1, \ldots, M$, of income distribution. But the demand functions that satisfy the integrability conditions corresponding to the functions of income distribution are generated by some Bergson function. A "party program" which does not satisfy the integrability conditions disorganizes the economical system, and cannot be considered as constructive. Economical agents with rational behavior would not support such a program.

If all the social groups agree with a program, then Eq. (2) generates the distribution of income, which ensures that the integrability conditions are satisfied for ultimate demand functions.

Finally, a social agreement generates economic structures which ensures self-organization of the economic agents, allows the cost law to hold, and makes financial regulating mechanisms maximally effective.

\section{NONPARAMETRIC METHOD FOR ANALYZING BUDGET STATISTICS}

To construct numerically the Bergson function, we need initial information. Usually, this is the budget statistics $\left\{X^{t, \alpha}, \mathbf{p}^{t} \mid t=1, \ldots, T ; \alpha=1, \ldots, M\right\}$, where $\mathbf{p}^{t}$ are prices at the time period $t$, and $X^{t, \alpha}$ is the consumption vector of the $\alpha$ th social group at the time period $t$. Applying the nonparametric method for constructing economical indices (represented in the report by the "trade statistics" $\left\{X^{t, \alpha}, \mathbf{p}^{t} \mid t=\right.$ $1, \ldots, T ; \alpha=1, \ldots, M\}$ of the $\alpha$ th social group, we can construct the price index $q_{\alpha}(\mathbf{p})$ from the point of view of the $\alpha$ th social group. Putting $u_{\alpha_{t}}^{t}=\frac{\left(\mathbf{p}^{t}, X^{t, \alpha}\right)}{q_{\alpha}(\mathbf{p})}$, and applying the nonparametric method, $\left\{q\left(\mathbf{p}^{t}\right), u^{t} \mid t=1, \ldots, T ; u^{t}=\left(u_{1}^{t}, u_{2}^{t}, \ldots, u_{M^{t}}^{t}\right)\right\} \quad$ considered as trade statistics, we obtain the consumption index which is just the Bergson function generating the observed distribution of income. Thus the developed methods can be applied for analyzing budget statistics. 
The obtained results explain the Bergson function in a new way. Now it not only formalizes a normative concept of fair income distribution, but also characterizes the real distribution of income existing in the society. If real distribution of income between social groups can be described by means of a Bergson function, then the consumption of the society as a whole can be characterized by one index, and the price level can be characterized by the price index. In this case economy is organized well, and financial mechanisms regulate effectively the distribution of resources. On the other hand, this implies that social groups achieved a compromise in distributing income.

We can also try to find a subset of social groups whose distribution of income can be described by its own Bergson function. If so, a compromise is achieved between social groups of this subset. Then the latter can be considered as a single group and characterized by one consumption index and the corresponding price index. Thus we can construct trees of social groups which characterize correctly the social structure of the society. Studying such social structures is of separate interest in making social and political decisions. 


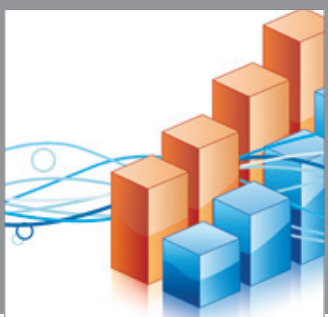

Advances in

Operations Research

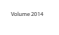

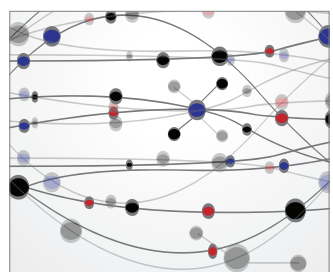

\section{The Scientific} World Journal
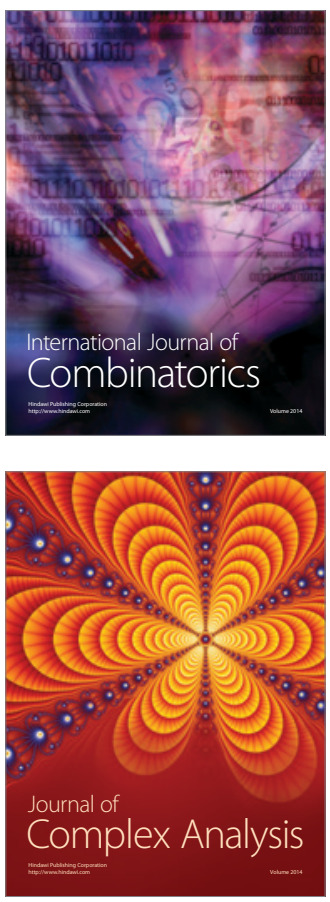

International Journal of

Mathematics and

Mathematical

Sciences
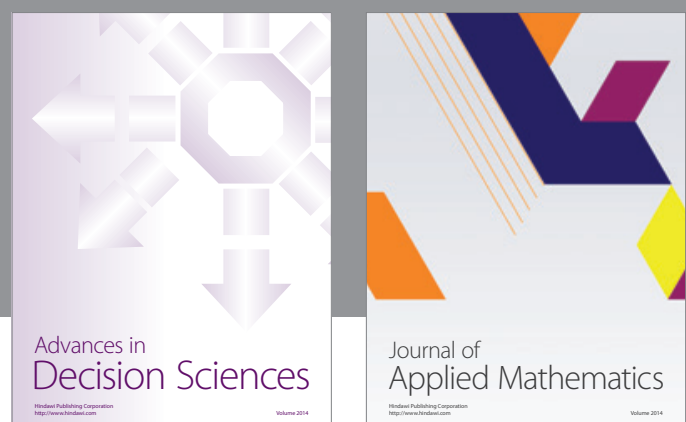

Journal of

Applied Mathematics
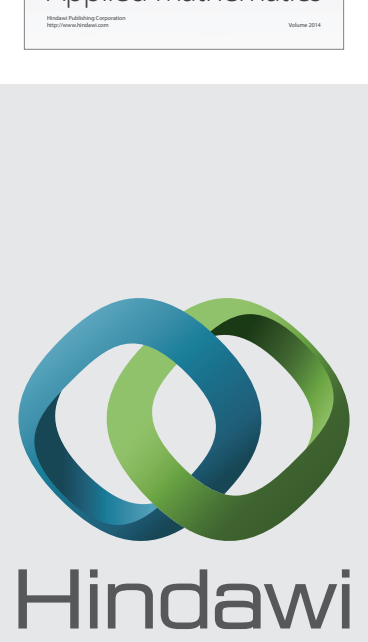

Submit your manuscripts at http://www.hindawi.com
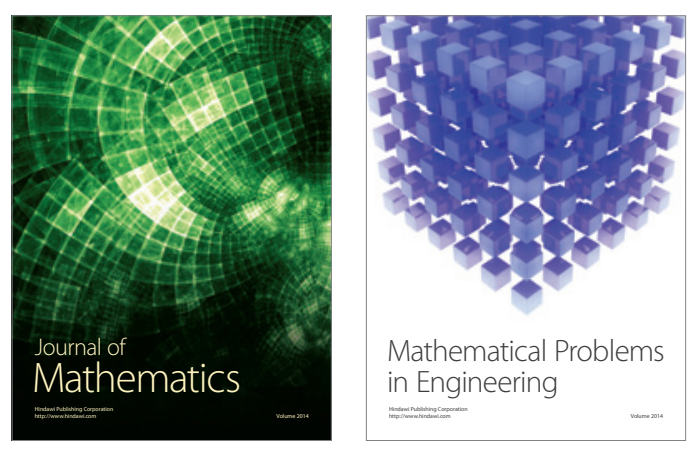

Mathematical Problems in Engineering
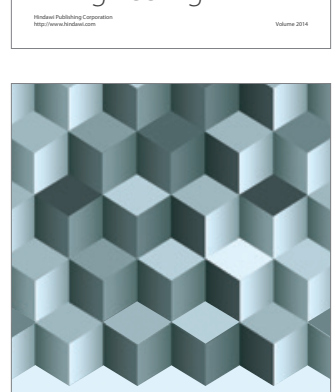

Journal of

Function Spaces
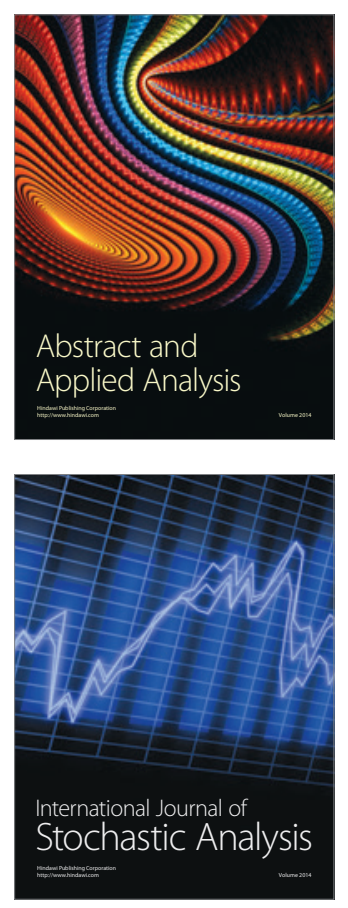

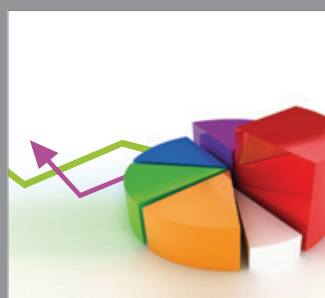

ournal of

Probability and Statistics

Promensencen
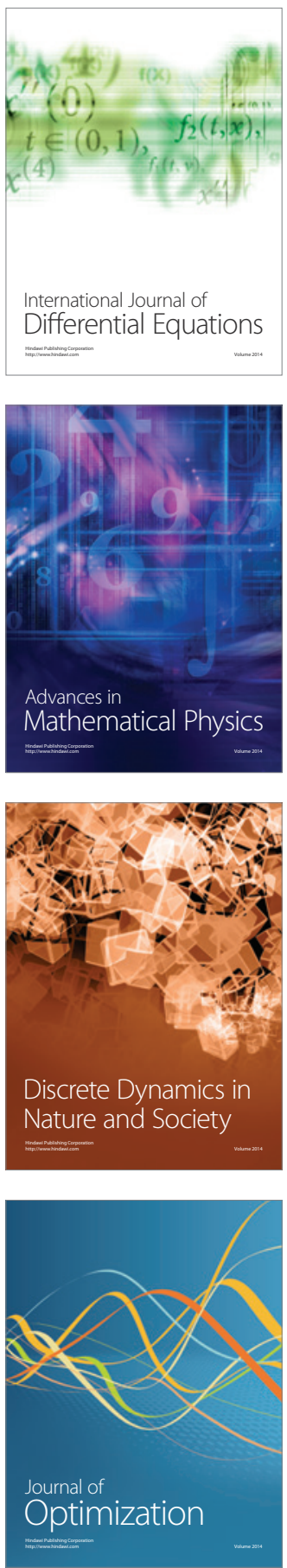